\author{
Andrea Alberti \\ Giovanni Mazzotta \\ Francesca Galletti \\ Paola Sarchielli
}

\section{Electroencephalographic brain mapping and migraine}

A. Alberti $\left({ }^{凶}\right) \cdot$ G. Mazzotta • F. Galletti P. Sarchielli

Department of Neuroscience,

University of Perugia, Perugia, Italy

e-mail: andrea_alberti@hotmail.com

Tel.: +39-075-5783609

Fax: $+39-075-5783870$

\begin{abstract}
Several studies have investigated brain bioelectrical activity with the aid of electroencephalographic brain mapping in migraine patients examined in the interictal period or during a spontaneous attack. The principal finding obtained between attacks was an increase in the power of the rapid activities, particularly in the range of the beta bands and especially in the temporal regions, in migraine patients both with and without aura. Another frequent occurrence between attacks is an increase in the absolute and relative powers of the slow theta and delta bands, which can be focal or diffuse. In our experience, the increase in the relative power of the theta band was prevalent bilaterally in the temporal-parietal regions in migraineurs with and without aura. Other authors detected interhemispheric asymmetries relative to alpha bands, more consistent in
\end{abstract}

patients with migraine with aura. During attacks the most relevant result is the decrease and asymmetry of dominant alpha activity, particularly on the side of the pain during attacks in patients with migraine without aura. In addition to changes in the alpha activities, an increase in the power of the slow delta and/or theta bands was found during attacks of migraine with aura. Although difficult to summarize in an integrated view, results of EEG brain mapping support the occurrence of bioelectrical abnormalities, which are present even between attacks and contribute towards defining the attack threshold, and which are accentuated near and during migraine crises as a consequence of neuronal events that underlie the pathogenic mechanisms of attacks.

Key words Electroencephalography • Brain mapping $\cdot$ Migraine

\section{Introduction}

Research from electroencephalographic examinations in patients with primary headaches has yielded controversial results. Some studies confirmed the presence of bio- electrical abnormalities in the electroencephalogram (EEG) in primary headache patients, particularly in migraine patients with and without aura. In contrast, other studies did not show abnormalities or any significant differences between headache patients and healthy control subjects. 
The picture of the findings available in the literature is therefore complex and it is difficult to formulate a coherent synthesis among discordant or dissimilar results. Lack of uniformity in the methodology used and different patient selection criteria could explain such contrasting results. Moreover, subjective visual analysis without EEG quantification limited the comparison between EEG findings. These limitations have been overcome by the advent of computerized and topographic EEG analysis, which has introduced new parameters of evaluation, allowing the representation of cerebral bioelectrical activity in a topographic system instead of the traditional EEG.

\section{Computerized and topographic EEG analysis}

The EEG can be elaborated through computer analysis procedures and represented in a topographic system called brain electrical activity mapping. Registration conditions are the same as those of classic electroencephalography. After the recording, parts of the trace to be analysed are accurately selected and an automatic rejection procedure is applied to suppress the bioelectrical abnormalities of extracerebral origin.

The computerized analysis consists of two main procedures: first, the analogue-to-digital conversion, and second, quantification of the EEG signals. The difference of potentials, as recorded by the electrodes, is an analogue signal, which has to be converted into digital form in order to be elaborated through quantitative analysis. Spectral analysis is the most diffuse technique for EEG quantification. This procedure is based on signal representation of the frequency domain, rather than of the time domain as occurs in classic electroencephalography. In particular, fast Fourier transform (FFT) is used to analyse the trace frequency composition. The signal is first processed by FFT and is then converted into sinusoidal waves with frequency in delta, theta, alpha and beta bands. In this way, new evaluation parameters are introduced, such as spectrum power, which represents how the power, i.e. the energy signal per unit of time, varies as a function of the frequency. For each band, it is possible to obtain the value of absolute power by summing all contributing frequency components. In contrast, relative power expresses the percentage of one band power with respect to the summed percentages of the other bands.

Data obtained through spectral analysis can be reproduced in a map, consisting of a bi-dimensional topographic system that substitutes the traditional trace in visualizing brain bioelectrical activity. Furthermore, the data from these parameters can be elaborated through statistical analysis and represented in a significance probability mapping.

Computerized analysis of the EEG allows brain electrical activity to be represented in a different manner - even small electroencephalographic alterations can be detected with respect to the visual subjective analysis - and allows intra- and interindividual comparisons to be carried out using statistically significant parameters.

\section{EEG mapping in migraine between attacks}

Several authors applied EEG mapping to migraine patients who were assessed during the interictal period or in the course of a spontaneous attack. The principal finding obtained between attacks was the increase in the power of the rapid activities, particularly in the range of the beta bands [1] and especially in the temporal regions, both in migraine patients with aura (70\% of cases) and without aura [2].

Another frequent occurrence during the intercritical phase is an increase in the absolute and relative powers of the slow bands, theta and delta, which can be focal or diffuse, depending on the different patient populations studied.

Lia et al. [3] demonstrated in $39.3 \%$ of the patients examined (17 with migraine without aura and 11 with migraine with aura) an increase in the relative power of theta and delta activity bilaterally in the parietal-occipital regions, with a reduction in the power of the alpha rhythm compared with control subjects. Similar results were obtained by Guidetti et al. [4], but they were unilaterally located and consistent with the side of pain in the majority of attacks.

In the study by Neufeld et al. [5], significant differences emerged only between control subjects and patients with migraine with aura. These consisted of a decrease in the absolute power peak of the alpha band in the latter group. Analogous findings were obtained by Polich et al. [6], who also found an increase in theta band power in the central-parietal regions.

Other authors detected interhemispheric asymmetries concerning the alpha bands. In a study carried out by Jonkman and Lelieveld [7], almost $55 \%$ of patients showed the above asymmetries in the alpha band, compared with $5 \%$ of control subjects, in terms of absolute power (55\% of cases) and peak frequency (20\% of cases).

The finding of an asymmetry in the alpha band is probably less constant in patients with migraine without aura than in those with migraine with aura. In fact, Facchetti et al. [8] found an asymmetry in alpha absolute power in $41.9 \%$ of patients with migraine with aura, whereas in $54.8 \%$ there was an asymmetry of the peak frequency. 


\section{EEG mapping during migraine attacks}

Several EEG mapping studies recorded during spontaneous migraine attacks reported that bioelectrical abnormalities mostly regarded alpha activity. In particular, the study of De Tommaso et al. [9] showed a decrease and an asymmetry of dominant alpha activity on the side of pain during attacks of migraine without aura compared with the headache-free period. The reduction in alpha activity on the headache side can be detected even 18-12 $\mathrm{h}$ before migraine attack onset. This phenomenon could account for the interhemispheric activity asymmetry observed in the interictal period [10].

The temporal relationship between attacks and asymmetry of bioelectrical activity is complex. In this regard, Nyrke et al. [11] carried out several EEG recordings at different time intervals relative to the crises and found an increase in bioelectrical abnormalities mostly concerning the alpha band in the period immediately preceding the attacks compared to 10 days before.

In addition to the changes in alpha activities, an increase in the power of the slow bands, delta and/or theta, was observed during attacks of migraine with aura [12, 13].

\section{Our experience with EEG mapping}

With the aim of investigating the electroencephalographic alterations in migraine patients during the headache-free period by the use of a computerized and topographic EEG analysis, 27 migraine patients attending the Headache Centre of the Neuroscience Department, University of Perugia, were examined: 15 patients with migraine without aura (mean age, $34.3 \pm 8.9$ years) and 12 with migraine with aura (mean age, 33.6 \pm 11.4 years). All patients with migraine with aura reported visual symptoms and six of them also paresthesias. No patient was affected by other neurological disorders or was in prophylactic treatment for migraine. The control group included 22 apparently healthy volunteers (mean age, 32.6 \pm 12.2 years) not suffering from headache or other neurological disorders.

Patients were studied at least $72 \mathrm{~h}$ from the last attack. The EEG recordings were made with the subjects at rest in a quiet, comfortable room. Nineteen electrodes were placed on the scalp according to the "International 10-20 System". Computerized analysis was made on chosen artefact-free periods for at least $2 \mathrm{~min}$. The signal was processed by FT and the absolute power spectrum values of delta, theta, alpha and beta bands were obtained. The $t$-test was used for the mean comparison of all parameters between patients and control subjects and between the subgroups of patients with migraine with and without aura.

Migraine patients showed a statistically significant increase in the relative power of the theta band, bilateral$1 y$, in the temporal-parietal regions $(p<0.05)$. No significant differences were found among relative power values between patients with migraine with aura and those without aura for each frequency band.

\section{Conclusions}

EEG findings concur with other neurophysiological experiences indicating migraine as a condition characterized by an altered neuronal excitability due to a peculiar biochemical milieu favouring a greater susceptibility to depolarization. These alterations are also present in the interictal period and could reflect the bioelectrical threshold for migraine attacks. They tend to accentuate immediately before and during migraine attacks, but the underlying mechanisms still need to be elucidated.

\section{References}

1. Robbins L, Northbrook IL (1990) Brain mapping in headache patients: lack of clinical utility. Headache 30:247

2. Hughes JR, Robbins LD (1990) Brain mapping in migraine. Clin Electroencephalogr 21:14-24

3. Lia C, Carenini L, Degioz C, Bottacchi E (1995) Computerized EEG analysis in migraine patients. Ital J Neurol Sci $16: 249-254$
4. Guidetti V, Seri S, Cerquiglini A, Brinciotti M (1989) Computerized EEG topography in childhood headache. Cephalalgia 28[Suppl 10]:190-192

5. Neufeld MY, Treves TA, Korczyn AD (1991) EEG and topographic frequency analysis in common and classic migraine. Headache 31:232-236

6. Polich J, Ehlers CL, Dalessio DJ (1986) Pattern-shift visual evoked responses and EEG in migraine. Headache 26:451-456
7. Jonkman EJ, Lelieveld MH (1981) EEG computer analysis in patients with migraine. Electroencephalogr Clin Neurophysiol 52:652-655

8. Facchetti D, Marsile C, Faggi L, Donati E, Kokodoko A, Poloni M (1990) Cerebral mapping in subjects suffering from migraine with aura. Cephalalgia 10:279-284 
9. De Tommaso M, Sciruicchio V, Guido M, Sasanelli G, Specchio LM, Puca FM (1998) EEG spectral analysis in migraine without aura attacks. Cephalalgia 18:324-328

10. Schoenen J, Thomsen LL (2000) Neurophysiology and autonomic dysfunction in migraine. In: Olesen J, TfeltHansen P, Welch KMA (eds) The headaches, 2nd edn. Lippincott Williams \& Wilkins, Philadelphia, pp 301-312
11. Nyrke T, Kangasniemi P, Lang H (1990) Alpha rhythm in classical migraine (migraine with aura): abnormalities in the headache-free interval. Cephalalgia 10:177-181

12. Sand T (1998) QEEG in migraine without aura. Cephalalgia 18:303
13. Puca FM, Minervini MG, Genco S, Savarese MA, Antonaci F, Brancasi B, Napoletano V, Monetti C (1985) EEG spectral analysis in common and classic migraine. Cephalalgia 5[Suppl 3]:382 